\title{
High-Fat Diet Augments VPAC1 Receptor-Mediated PACAP Action on the Liver, Inducing LAR Expression and Insulin Resistance
}

\author{
Masanori Nakata, ${ }^{1}$ Boyang Zhang, ${ }^{1}$ Yifei Yang, ${ }^{1}$ Takashi Okada, ${ }^{2}$ Norihito Shintani, ${ }^{3}$ \\ Hitoshi Hashimoto, ${ }^{3,4,5}$ and Toshihiko Yada $^{1}$ \\ ${ }^{1}$ Department of Physiology, Division of Integrative Physiology, Jichi Medical University School of Medicine, \\ Shimotsuke, Tochigi 329-0498, Japan \\ ${ }^{2}$ Department of Biochemistry and Molecular Biology, Division of Gene Therapy, Research Center for Advanced Medical Technology, \\ Nippon Medical School, Tokyo 113-8603, Japan \\ ${ }^{3}$ Laboratory of Molecular Neuropharmacology, Graduate School of Pharmaceutical Sciences, Osaka University, \\ Suita, Osaka 565-0871, Japan \\ ${ }^{4}$ Molecular Research Center for Children's Mental Development, United Graduate School of Child Development, \\ Osaka University, Kanazawa University, Hamamatsu University School of Medicine, Chiba University and University of Fukui, \\ Suita, Osaka 565-0871, Japan \\ ${ }^{5}$ Division of Bioscience, Institute for Datability Science, Osaka University, Suita, Osaka 565-0871, Japan
}

Correspondence should be addressed to Toshihiko Yada; tyada@jichi.ac.jp

Received 17 August 2016; Revised 26 October 2016; Accepted 13 November 2016

Academic Editor: Pedro M. Geraldes

Copyright ( $) 2016$ Masanori Nakata et al. This is an open access article distributed under the Creative Commons Attribution License, which permits unrestricted use, distribution, and reproduction in any medium, provided the original work is properly cited.

\begin{abstract}
Pituitary adenylate cyclase-activating polypeptide (PACAP) acts on multiple processes of glucose and energy metabolism. PACAP potentiates insulin action in adipocytes and insulin release from pancreatic $\beta$-cells, thereby enhancing glucose tolerance. Contrary to these effects at organ levels, PACAP null mice exhibit hypersensitivity to insulin. However, this apparent discrepancy remains to be solved. We aimed to clarify the mechanism underlying the antidiabetic phenotype of PACAP null mice. Feeding with high-fat diet (HFD) impaired insulin sensitivity and glucose tolerance in wild type mice, whereas these changes were prevented in PACAP null mice. HFD also impaired insulin-induced Akt phosphorylation in the liver in wild type mice, but not in PACAP null mice. Using GeneFishing method, HFD increased the leukocyte common antigen-related (LAR) protein tyrosine phosphatase in the liver in wild type mice. Silencing of LAR restored the insulin signaling in the liver of HFD mice. Moreover, the increased LAR expression by HFD was prevented in PACAP null mice. HFD increased the expression of VPAC1 receptor (VPAC1-R), one of three PACAP receptors, in the liver of wild type mice. These data indicate that PACAP-VPAC1-R signaling induces LAR expression and insulin resistance in the liver of HFD mice. Antagonism of VPAC1-R may prevent progression of HFD-induced insulin resistance in the liver, providing a novel antidiabetic strategy.
\end{abstract}

\section{Introduction}

Pituitary adenylate cyclase-activating polypeptide (PACAP) regulates glucose and energy metabolism through central and peripheral actions. PACAP potentiates glucose-induced insulin release from pancreatic islet and promotes insulinmediated glucose disposal in adipocyte [1-3]. In addition, in hunger and fasted conditions, PACAP increases food intake and catecholamine release to maintain the energy supply [4-6]. PACAP is localized in the central and peripheral tissues [7]. In the central nervous system (CNS), PACAP is localized abundantly in the hypothalamus [8] and in the brain stem, including the nucleus tractus solitarius (NTS) and dorsal motor vagal nucleus (DMV) [9], the areas that regulate energy metabolism. PACAP is also localized in a wide range of peripheral nerves including the sympathetic nervous 
system. Thus, PACAP is thought to act as a neurotransmitter or neuromodulator in the central and peripheral nerves.

There are three types of PACAP receptors: PAC1 receptor (PAC1-R) selective for PACAP [10], and VPAC1-R and VPAC2-R shared by PACAP and VIP with equal potencies [11]. Among three types of PACAP receptors, PAC1-R is expressed in pancreatic islets and VPAC2-R in pancreatic islets and adipocyte $[12,13]$. PACAP enhances glucosestimulated insulin secretion in islets and insulin-induced glucose uptake in adipocytes, the effects acting as antidiabetic [1-3]. Contradictory to these actions, PACAP null mice reportedly exhibit increased insulin sensitivity with regular chow diet [14]. Moreover, PACAP null mice are resistant against high-fat diet- (HFD-) induced insulin resistance. These results indicate that PACAP might impair the insulin action in the tissues other than adipose tissue. The liver is one of the major organs that are targeted by insulin and implicated in insulin resistance, and it expresses PACAP receptors. It was previously reported that PACAP reportedly stimulates adenylate cyclase in hepatocytes and glucose output from them $[15,16]$. Hence, the liver could be the candidate target for the PACAP action on glucose metabolism. In this study, we aimed to elucidate the signal mechanism and function of PACAP in the liver of mice fed HFD.

\section{Materials and Methods}

2.1. PACAP Null Mice and Wild Type Mice. The generation and maintenance of the PACAP null mice on the ICR background have been previously described in detail [4]. Fiveweek-old male ICR mice and C57BL/6J mice were obtained from Japan SLC (Hamamatsu, Japan). In this study, PACAP null mice and wild type ICR mice were single-housed under a $12 \mathrm{~h}$ light : $12 \mathrm{~h}$ dark cycle (lights on at 07:30 a.m.) conditions. Mice were randomly divided into two groups fed with either a regular chow or a HFD for 6 weeks. In the chow (CE-2; CLEA, Japan), $58.9 \%$ of calories was derived from carbohydrate and $11.6 \%$ of calories was derived from fat, yielding total calories of $344 \mathrm{kcal} / 100 \mathrm{~g}$. In the HFD (Quick fat CLEA-Japan, Tokyo, Japan), $44.6 \%$ of calories was derived from carbohydrate containing sucrose and $32.3 \%$ of calories was derived from fat, $425 \mathrm{kcal} / 100 \mathrm{~g}$. Experimental procedures and care of animals were carried out according to the Jichi Medical University Institute of Animal Care and Use Committee.

2.2. Glucose Tolerance and Insulin Tolerance Tests. For glucose tolerance tests (GTT), mice at 6 weeks after regular chow and HFD fed were fasted 6-h. Following administration of glucose ( $2 \mathrm{~g}$ per $\mathrm{kg}$ i.p.), glucose levels were measured in tail blood at indicated time points. For insulin tolerance tests (ITT), mice were fasted 6-h. Following administration of insulin (0.5 IU per kg i.p.), glucose levels were measured in tail blood at indicated time points.

2.3. GeneFishing Analysis of Mouse Liver RNA Expression. $\mathrm{C} 57 \mathrm{BL} / 6 \mathrm{~J}$ mice at six weeks old were randomly assigned to one of two different dietary groups for 6 weeks $(n=4)$. At 6 weeks after regular chow or HFD fed, total RNA of liver was isolated using TRIzol (Invitrogen, Carlsbad, CA) and treated with RQ1-DNase (Promega, Madison, WI) to remove residual contaminations with DNA. To analyze the difference of RNA expression of both group mice liver, we used a new differential display PCRs method that is based on annealing control primers (ACPs). Reverse transcription was conducted using the GeneFishing ${ }^{\mathrm{TM}}$ DEG kits (Seegene, Seoul, Korea) as follows; $3 \mu \mathrm{g}$ of total RNA was converted to cDNA using ReverTra Ace (Toyobo, Osaka, Japan) with dT-ACP1 primer. The synthesized first-strand cDNA samples were subjected to PCR amplification using dT-ACP2 and one of arbitrary ACPs as primer. After incubation at $94^{\circ} \mathrm{C}$ for $5 \mathrm{~min}, 50^{\circ} \mathrm{C}$ for $3 \mathrm{~min}$, and $72^{\circ} \mathrm{C}$ for $1 \mathrm{~min}, 40$ cycles of $94^{\circ} \mathrm{C}$ for $40 \mathrm{sec}, 65^{\circ} \mathrm{C}$ for $40 \mathrm{sec}$, and $72^{\circ} \mathrm{C}$ for $40 \mathrm{sec}$ followed, after which a postextension was performed at $72^{\circ} \mathrm{C}$ for $5 \mathrm{~min}$. The PCR products were then subjected to electrophoresis on a $2 \%$ agarose gel and stained with $1 \mathrm{mg} / \mathrm{mL}$ ethidium bromide. Amplified PCR products were cloned into a pGEM-T vector using the TA cloning system (Takara Bio, Ohtsu, Japan) for further analysis. Double-stranded DNA was sequenced by Hitachi model SQ-5500 DNA auto-sequencer by using delta Taq fluorescent dye-primer cycle sequencing kit (Amersham Corp., Arlington Heights, IL) and Texas-red labelled primer.

2.4. Construction of Short Hairpin RNAs (shRNAs) and Viral Vector Production. Target sequence for mouse LAR was chosen to design shRNAs (accession number NM 011213.2: $5^{\prime}$ CGAATTACGTGGATGAAGA-3' .530 to 548). In addition, scrambled oligonucleotide sequence was used for specificity control (5'-CAACACTAGTTGACATGTA- $\left.3^{\prime}\right)$. The mU6 promoter, hairpin sequence and terminator sequences were subcloned into pAAV plasmid. The shRNA constructs were then cloned into an AAV serotype 9 (AAV9) for stable shRNA delivery. Briefly, AAV9-LAR-shRNA and AAV9-ScrshRNA viruses were produced following triple-transfection of HEK293 cells with pAAV-shRNA, an adenoviral helper plasmid pAdeno, and a chimeric helper plasmid encoding AAV2 rep/AAV9 cap genes (pAAV2rep/AAV9cap). The purification and titration of AAV were reported as previously described [17].

2.5. Administration of $A A V$ Vectors In Vivo. For intraliver administration, vectors were dissolved in $100 \mu \mathrm{L}$ saline and injected directly into the portal vein of the mice. AAV-LARshRNA or control AAV-scrambled-shRNA $\left(1 \times 10^{12} \mathrm{vg}\right)$ was administered to 6 weeks HFD fed C57BL/ 6 mice. At 1 weeks after administration of AAV vectors, insulin tolerance test and measurement of the insulin receptor phosphorylation in liver of both group mice were performed.

2.6. Western Blot. Phosphorylation of Akt and insulin receptor was measured by western blot as previously reported [18]. After intraperitoneal administration of insulin (0.5 IU per $\mathrm{kg}$ i.p.), livers were removed and lysed in $1 \mathrm{~mL}$ lysis buffer containing $100 \mathrm{mM}$ sodium fluoride, $10 \mathrm{mM}$ sodium $\mathrm{PPi}$, and $2 \mathrm{mM}$ sodium orthovanadate. The $10 \mu \mathrm{g}$ proteins were subjected to $8 \%$ SDS-PAGE and transferred to nitrocellulose 
filters. Phosphorylation of Akt and insulin receptor were detected with the polyclonal antibodies, phospho-Ser473 Akt (CST, Beverly, MA), or phosphor-Tyr1361 insulin receptor $\beta$-subunit (Abcam plc, Cambridge, UK). Immunoreactive proteins were detected with HRP-conjugated secondary antibody and the ECL system (Amersham Corp., Arlington Heights, IL). After stripping, membrane was hybridized with anti-Akt antibody (CST, Beverly, MA) or anti-insulin receptor $\beta$-subunit (Santa Cruz bio., Santa Cruz, CA). Then, immunoreactive signal was quantified by FAS-1000 (Fujifilm, Japan) and signal levels of phosphor-Akt and phosphorinsulin receptor were normalized to Akt and insulin receptor $\beta$-subunit.

Liver lysate proteins of mice administrated of AAV vectors were subjected to $8 \%$ SDS-polyacrylamide gel electrophoresis and transferred to nitrocellulose filters. LAR proteins were detected with the anti-LAR IgG (Transduction Lab, Lexington, KY) and ECL system. Immunoreactive signal was quantified by using FAS-1000 (Fujifilm, Tokyo, Japan) and expression levels of proteins were normalized to $\beta$-actin (Santa Cruz bio.).

2.7. Measurements of $m R N A$. Quantification of mRNA expressions measured by real-time PCR has been previously described in detail [19]. Total RNA was isolated using Trizol and RNase-free DNase. After the conversion to cDNA, realtime PCR was performed with SYBR premix Ex taq II polymerase (Takara Bio). Expression levels were calculated by the $\Delta \Delta C T$ method. The primers used in this experiment were listed in Supplemental Table 1 in Supplementary Material available online at http://dx.doi.org/10.1155/2016/9321395.

2.8. Liver Slice Culture. Transverse liver slices were prepared from 5-week-old male ICR mice as previously reported [20]. Liver was isolated from anesthetized mice, from which slices of $300 \mu \mathrm{m}$ thickness were prepared with a vibratome. Tissue slices were explanted on a culture membrane (Millicell $\mathrm{CM}$, pore size $0.4 \mu \mathrm{m}$, Millipore) in a $35 \mathrm{~mm}$ Petridish sealed with Parafilm (American Can Co., Greenwich) and cultured with test reagents in $1.0 \mathrm{~mL}$ DMEM (GibcoInvitrogen), which was supplemented with $2.7 \mathrm{mM} \mathrm{NaHCO}_{3}$, $10 \mathrm{mM}$ HEPES, $20 \mathrm{mg} / \mathrm{L}$ kanamycin (Gibco), $100 \mathrm{mg} / \mathrm{mL}$ apo-transferin (Sigma), $5 \mu \mathrm{g} / \mathrm{mL}$ insulin (Sigma), $100 \mathrm{~mm}$ putrescine (Sigma), $20 \mathrm{nM}$ progesterone (Sigma), and $30 \mathrm{nM}$ sodium selenite (Gibco) and incubated in $5 \% \mathrm{CO}_{2}$ at $37^{\circ} \mathrm{C}$ for overnight. After incubation for 24 hours, total RNAs were extracted and RT-PCR was performed as described earlier.

2.9. Statistical Analysis. All data are expressed as mean \pm sem. All data were subjected to one-way or two-way ANOVA. Differences between experimental groups were determined by the Tukey test. Statistical significance was accepted when $p<0.05$.

\section{Results}

3.1. PACAP Null Mice Were Protected against HFD-Induced Insulin Resistance. Mice fed regular chow or HFD for 6 weeks were subjected to GTTs. Under regular chow fed conditions, PACAP null mice, compared to wild type mice, exhibited a remarkably suppressed rise of blood glucose and quicker return to the basal level at $60 \mathrm{~min}$ (Figure 1(a)). Under HFD fed conditions, blood glucose was elevated to high levels over $400 \mathrm{mg} / \mathrm{dL}$ in wild type mice, which was markedly ameliorated in PACAP null mice (Figure 1(b)). In ITTs, under regular chow fed conditions, PACAP null mice exhibited the greater insulin sensitivity compared to wild type mice (Figure 1(c)). Furthermore, the severe insulin resistance induced by HFD was ameliorated in PACAP null mice (Figure 1(d)). These results indicate that PACAP deficiency promotes insulin sensitivity under regular chow fed conditions and counteracts insulin resistance induced by HFD.

To explore the mechanism for the enhanced insulin sensitivity in PACAP null mice, the phosphorylation (Ser473) of Akt, an insulin signaling molecule, in the liver was examined. Liver tissue samples were obtained from anaesthetized mice at $15 \mathrm{~min}$ after i.p. insulin injection ( $0.5 \mathrm{IU} / \mathrm{kg}$ body weight). Insulin was marked by phosphorylated Akt in the liver of wild type and PACAP null mice fed regular chow (Figure 2). Under HFD fed conditions, in contrast, Akt phosphorylation was markedly reduced in wild type mice, and this change was almost completely prevented in PACAP null mice, while total Akt protein content remained unchanged (Figure 2). These results indicate that PACAP counteracts the action of HFD to impair insulin-induced Akt phosphorylation in the liver.

3.2. The Expression of LAR in Liver Was Elevated in Mice Fed HFD. To explore the molecules induced by HFD, the differences in mRNA expression in the liver between regular chow and HFD conditions were analyzed using GeneFishing. In the liver of mice fed HFD, 21 elevated genes and 4 reduced genes were identified. One elevated gene was leukocyte common antigen-related (LAR) protein tyrosine phosphatase, also known as receptor type protein tyrosine phosphatase F (PTPRF), which is involved in insulin signaling [15]. Quantitative PCR also showed that HFD treatment markedly elevated LAR mRNA expression in the liver (Figure 3(a)). On the other hand, the elevated LAR mRNA expression in the liver under HFD conditions was significantly decreased in PACAP null mice (Figure 3(b)), suggesting that PACAP increases LAR mRNA expression under HFD conditions.

To assess the pathological link between overexpression of LAR and insulin resistance in HFD mice, we silenced LAR by using shRNA expression AAV vector. Intraportal administration of AAV-LAR-shRNA vector significantly reduced the expression of LAR in liver of HFD mice (Figures 4(a) and 4(b)). The treatment with LAR-shRNA, compared to ScrshRNA, ameliorated insulin resistance in HFD mice (Figures $4(\mathrm{a})$ and $4(\mathrm{c})$ ). Furthermore, the phosphorylation of insulin receptor $\beta$-subunits in the liver in AAV-LAR-shRNA administrated mice was significantly increased compared to that of control mice. AAV-LAR-shRNA administration significantly improved insulin sensitivity in HFD mice (Figure 4(d)). These results indicated that the overexpression of LAR in 


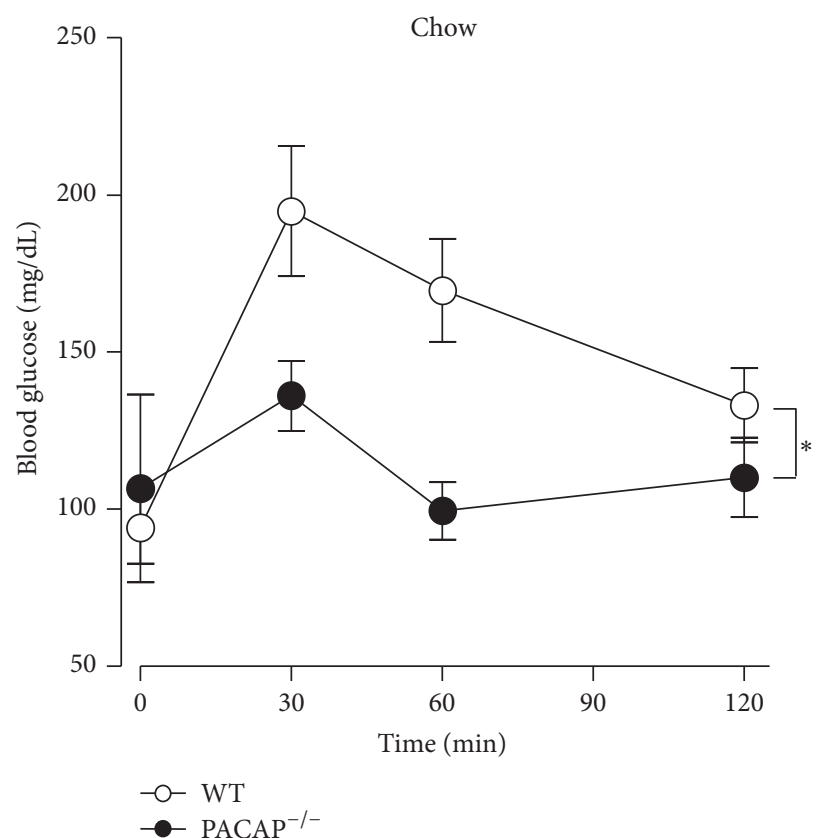

(a)

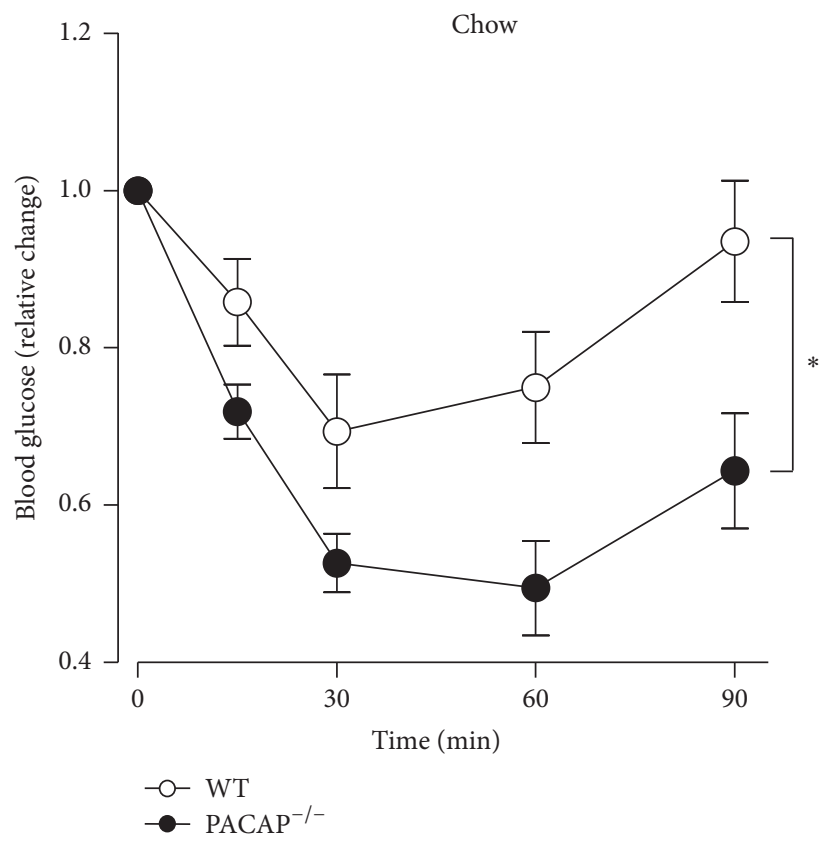

(c)

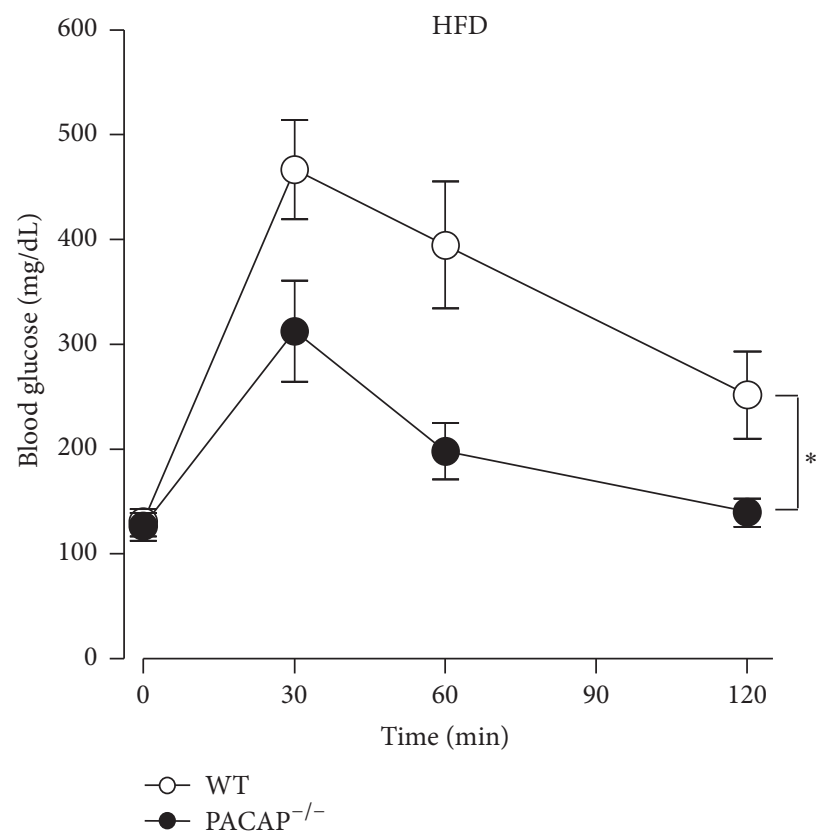

(b)

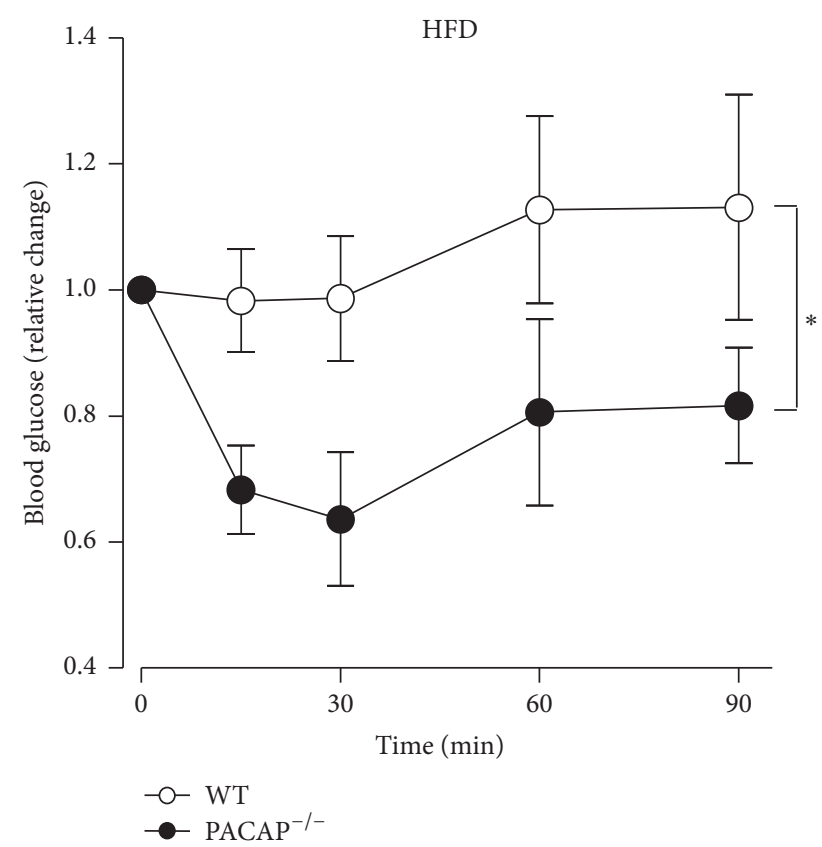

(d)

FIGURE 1: Effects of HFD on glucose tolerance and insulin tolerance in wildtype and PACAP null mice. (a) and (b) Blood glucose levels in GTT in wild type (WT) (open symbols) and PACAP null mice (filled symbols) fed regular chow (a) and HFD (b) for 6 weeks. Glucose at $2 \mathrm{~g} / \mathrm{kg}$ body weight was injected to mice fasted $6 \mathrm{~h}$. (c) and (d) Blood glucose levels in ITT in WT and PACAP null mice fed regular chow (c) and HFD (d) for 6 weeks. Insulin at $0.5 \mathrm{IU} / \mathrm{kg}$ body weight was injected to mice fasted for $6 \mathrm{~h}$. All data are presented as means \pm s.e.m. ${ }^{*} p<0.05$.

liver, which was induced by HFD through PACAP, impaired the insulin sensitivity in vivo.

This finding prompted us to examine direct effect of PACAP on LAR mRNA expression in the liver slices in vitro experiments. In the liver slices prepared from ICR mice and cultured in DMEM solution for $24 \mathrm{~h}$, the treatment with $10^{-8}$ M PACAP38 markedly increased LAR mRNA expression (Figure 5(a)). VIP also significantly increased LAR mRNA expression (Figure 5(b)). Both $10^{-9} \mathrm{M}$ PACAP and $10^{-10} \mathrm{M}$ TNF- $\alpha$ did not increase but coadministration significantly 


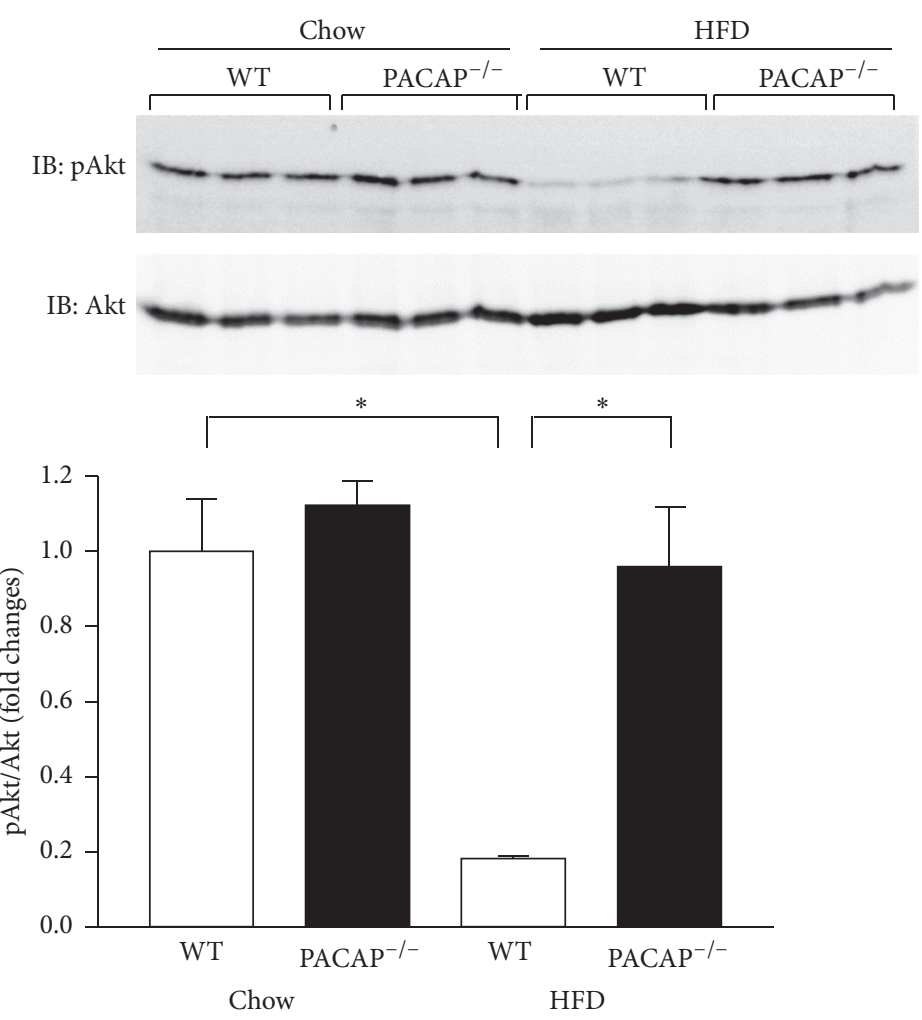

FIGURE 2: Insulin-induced Akt phosphorylation in liver. Western blot analysis using anti-phospho Akt and anti-Akt antibodies. Liver samples were collected form wild type (WT) and PACAP null $\left(\mathrm{PACAP}^{-/-}\right)$mice fed regular chow and HFD for 6 weeks. The intensity of Akt phosphorylation is expressed by the ratio of phosphorylated over total Akt proteins. $n=3$. All data are presented as means \pm s.e.m. ${ }^{*} p<0.05$.

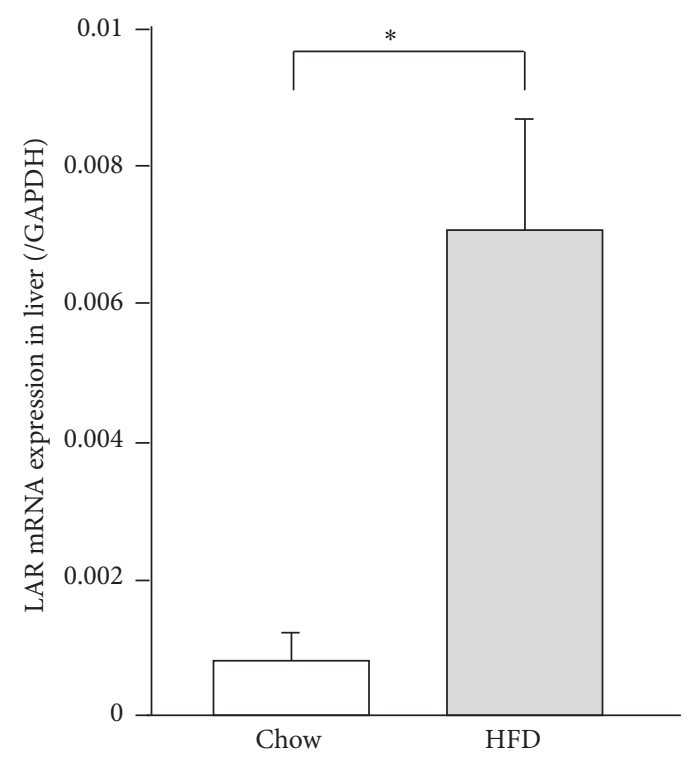

(a)

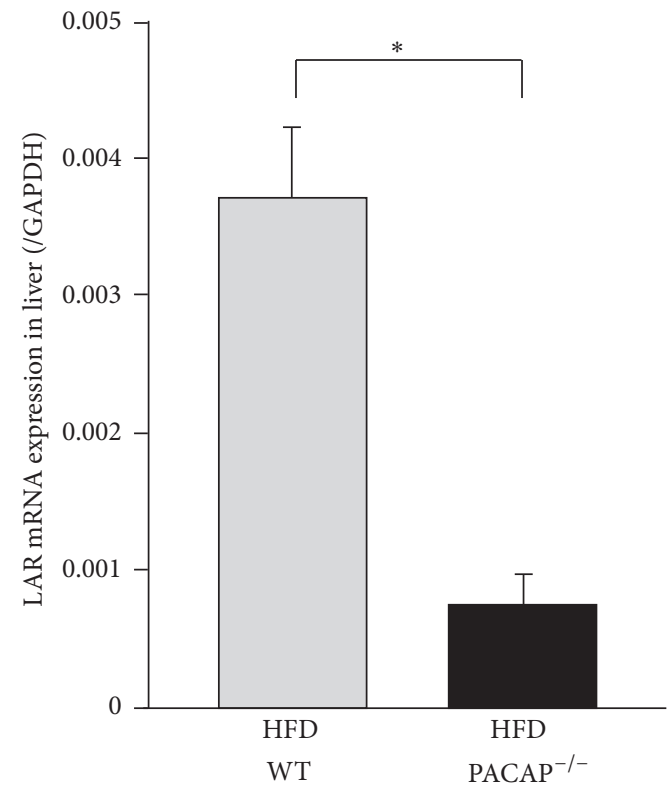

(b)

FIGURE 3: LAR expression in liver of mice. (a) LAR mRNA expression in the liver of regular chow fed and HFD fed ICR mice at 6 weeks. $n=5$ to 6 . (b) LAR mRNA expression in the liver of wild type and PACAP null mice fed HFD for 6 weeks. $n=5$ to 6 . All data are presented as means \pm s.e.m. ${ }^{*} p<0.05$. 


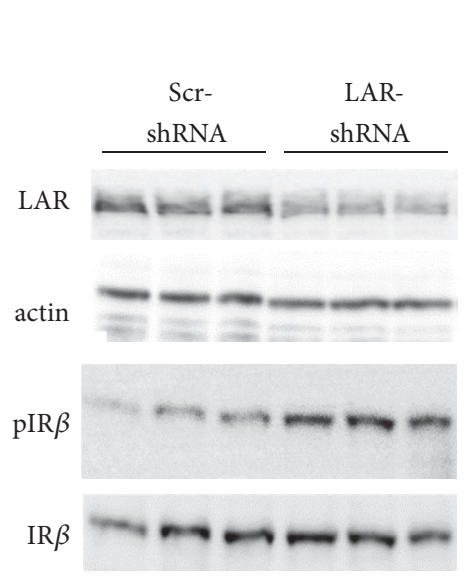

(a)

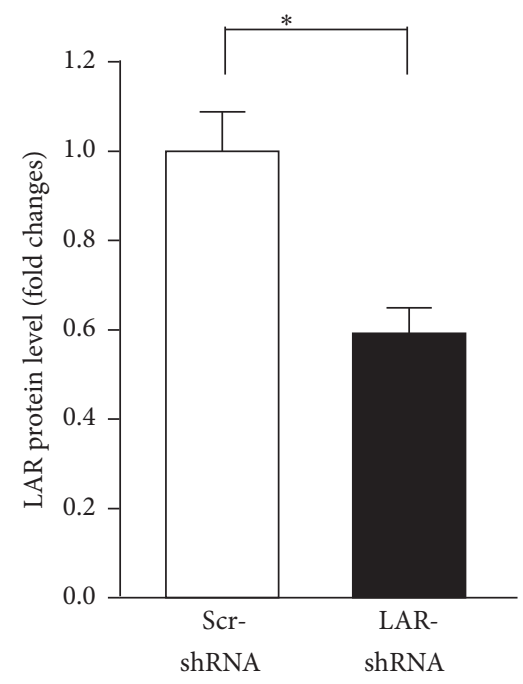

(b)

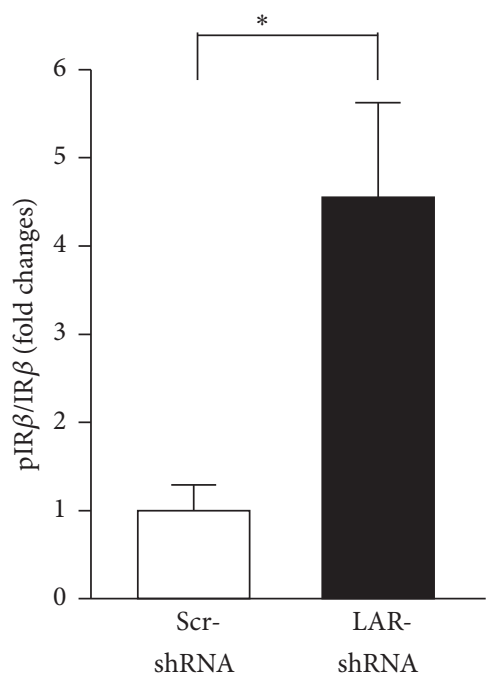

(c)

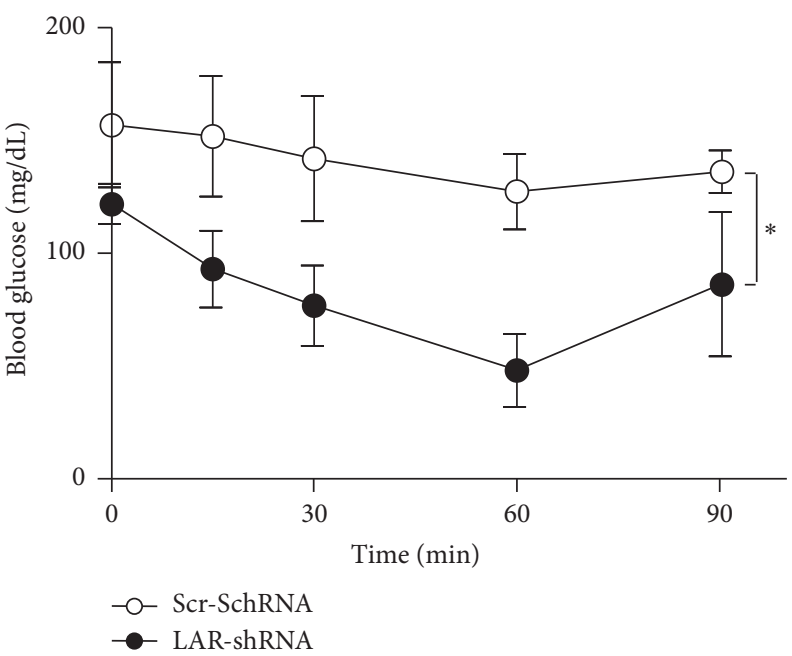

(d)

FIGURE 4: Effects of LAR knockdown on insulin sensitivity in liver of wild type mice fed HFD. (a) Western blot analysis using anti-LAR and anti-phospho-insulin receptor $\beta$-subunit $(\mathrm{pIR} \beta)$ in the liver of HFD mice at 1 week after injection of AAV-Scr-shRNA or LAR-shRNA. Liver samples were collected at $10 \mathrm{~min}$ after insulin injection $(0.5 \mathrm{IU} / \mathrm{kg}$ ). (b) LAR protein levels, relative to $\beta$-actin (actin), in the liver. $n=3$ for each group. (c) The intensity of IR $\beta$ phosphorylation is expressed by the ratio of phosphorylated over total insulin receptor $\beta$-subunit (IR $\beta$ ) proteins. (d) Blood glucose levels in ITT in HFD mice at 1 week after injection of AAV-Scr-shRNA or LAR-shRNA. Insulin at 0.5 IU/kg body weight was injected to mice fasted for $6 \mathrm{~h}$. All data are presented as means \pm s.e.m. ${ }^{*} p<0.05$.

increased LAR expression (Figure 5(c)). These results suggest that PACAP directly interacts with the liver to induce LAR mRNA expression.

3.3. VPAC1-R mRNA Expression Was Increased in Liver of Mice Fed HFD. The mRNA expressions of PACAP and three subclasses of PACAP receptors were examined. VAPC1-R at high levels and PAC1-R at low levels were expressed, while PACAP and VPAC2-R were not expressed, in the liver (Figure 6). The mRNA expression of VAPC1-R in the liver was 10 -fold elevated by HFD. These results suggest that under HFD conditions, the remarkable elevation of VPAC1-R may allow PACAP to induce LAR mRNA expression in the liver to induce insulin resistance.

\section{Discussion}

The present study aimed to clarify the mechanism underlying the antidiabetic phenotype of PACAP null mice. We found that HFD markedly increased LAR and VPAC1-R expressions in the liver, in parallel with systemic insulin resistance. Deficiency of PACAP counteracted the HFD-induced insulin resistance and elevation of LAR expression in the liver. Conversely, administration of PACAP increased LAR expression in the liver slices in vitro. The present study indicates that, 


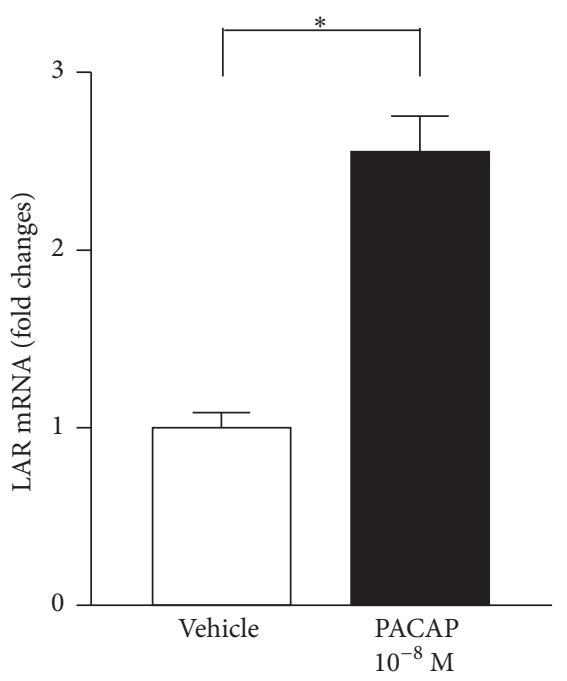

(a)

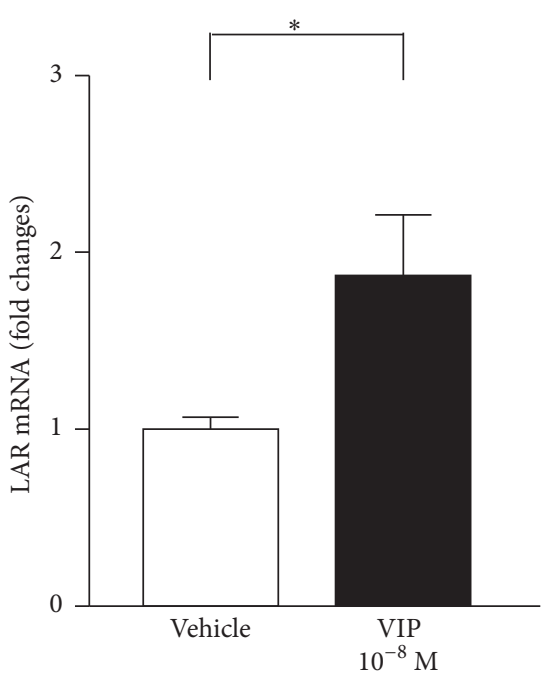

(b)

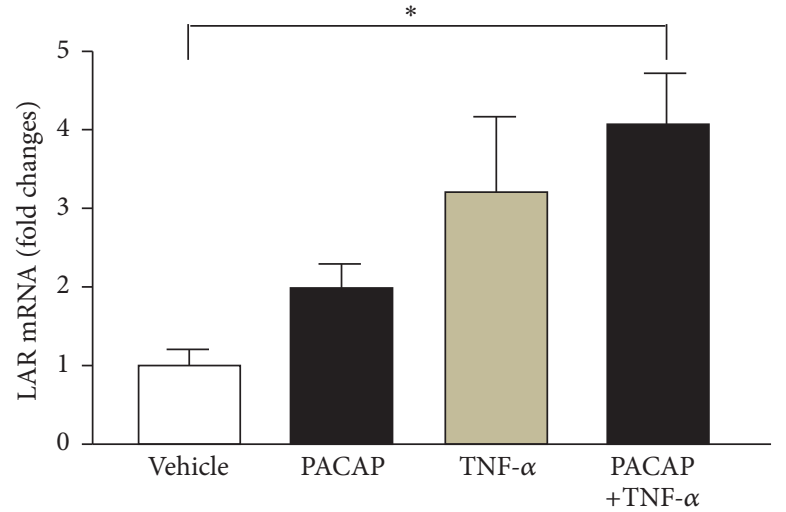

(c)

FIGURE 5: LAR expression in liver slice. (a) and (b) LAR mRNA expression in liver slices cultured with $10^{-8} \mathrm{M}$ PACAP (a) or $10^{-8} \mathrm{M}$ VIP (b) for $24 \mathrm{~h} . n=4$ for each group. (c) LAR mRNA expression in liver slices cultured with $10^{-9} \mathrm{M}$ PACAP and/or $10^{-10} \mathrm{M}$ TNF- $\alpha$ for $24 \mathrm{~h}$. $n=3$ for each group. Each bar represents mean \pm s.e.m. ${ }^{*} p<0.05$.

under HFD conditions, the remarkable elevation of VPAC1$\mathrm{R}$ may allow PACAP to induce LAR mRNA expression in the liver to induce insulin resistance.

In the present study, HFD-induced LAR expression in parallel with insulin resistance in the liver, while silencing of LAR expression in liver of HFD mice restored phosphorylation of insulin receptor. The activation of insulin receptor is triggered by its phosphorylation at tyrosine residues in response to insulin and terminated by dephosphorylation induced by protein tyrosine phosphatases (PTPs) [21-23]. Among PTPs, LAR is widely expressed in insulin-sensitive tissues and suppresses the insulin signaling in skeletal muscle and adipocyte [24, 25]. Moreover, deficiency of LAR upregulates $\mathrm{PI} 3 \mathrm{~K}$, an activator of Akt, in hepatoma cells [26]. Furthermore, LAR expression was elevated in liver of obese Zucker fatty rat [27]. Our results, combined with these reports, suggest that the elevated LAR in liver by HFD causes insulin resistance.
Chronic inflammation plays a key role in the pathogenesis of insulin resistance [28]. TNF- $\alpha$ promotes inflammation and suppresses insulin sensitivity in insulin target tissues including liver. TNF- $\alpha$ also increases LAR expression [27]. In our previous study, while plasma TNF- $\alpha$ level of PACAP null mice was not significantly different from that of wild mice, the LAR expression in liver was reduced in PACAP null mice, showing an apparent discrepancy [14]. We here demonstrated that PACAP potentiates the TNF- $\alpha$-induced increase in LAR expression. It is suggested that the potentiation of LAR expression by PACAP was abolished in PACAP null mice, resulting in counteraction of the insulin resistance.

In this study, PACAP was not detected in liver. Hence, the PACAP that interacts with VPAC1-R to elevate LAR may be derived from extraliver tissues. It was reported that PACAP is produced in both preganglionic and postganglionic sympathetic nerves and that PACAP-containing nerve fibers are observed in the gastrointestinal tract, adrenals, and 


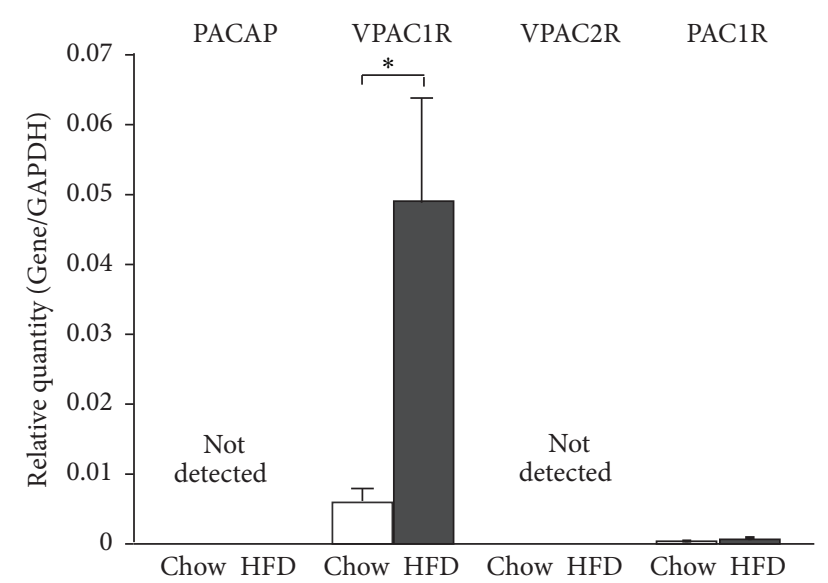

Figure 6: Expressions of PACAP and PACAR receptor subclasses in liver. The mRNA expressions of PACAP and PACAR receptors, VPAC1-R, VPAC2-R, and PAC1-R, in the liver of ICR mice fed regular chow and HFD for 6 weeks. $n=5-6$ for each group. Each bar represents mean \pm s.e.m. ${ }^{*} p<0.05$.

blood vessel walls in several organs [29-32]. Hence, PACAP could be derived from sympathetic nerves innervating the liver. It should also be noted that PACAP stimulates release of glucagon and catecholamines, the agents that stimulate adenylate cyclase activity in hepatocytes [33, 34]. Hence, PACAP could stimulate glucose output from hepatocytes not only via its direct action on the liver but also via releasing glucagon and/or catecholamine $[15,16]$.

The inhibitor of dipeptidyl peptidase IV (DPP-IV) is clinically used to treat type 2 diabetic patients. DPP-IV inhibitors lengthen the lifetime and hence promote the effect of antidiabetic hormones, glucagon-like peptide (GLP-1), and glucosedependent insulinotropic polypeptide (GIP). A big advantage of DPP-IV is that they induce pathologic hypoglycemia in a much smaller incidence than the classical antidiabetic drugs such as sulfonylureas [35]. The underlying mechanisms are not fully understood, though the glucose-dependent insulinotropic effects of GLP-1 and GIP are implicated. DPPIV plays a major role in the degradation of circulating PACAP38 [36, 37]. Hence, DPP-IV inhibitors are expected to lengthen the lifetime and promote the effect of PACAP. The present study suggests a possible mechanism that the promoted action of PACAP by DPP-IV inhibitors enhances glucose output from the liver to prevent hypoglycemia.

Several reports suggest a possible involvement of PACAP in development of type 2 diabetes, although this needs to be explored more in detail. PACAP in the subpicomolar range amplifies glucose-induced insulin release from islets [1,2], an action partly mediated by VPAC2-R [35]. VPAC2-R is located in the islets and adipocytes, and VPAC2-R specific agonists reportedly enhance insulin secretion and glucose disposal $[38,39]$. In addition, the present study found that VPAC1$\mathrm{R}$ in the liver is elevated and mediates insulin resistance in mice fed HFD. Collectively, the antagonism of VPAC1-R and agonism of VPAC2-R could work in concert to effectively counteract hyperglycemia [40]. Development of the selective
VPAC1-R antagonist may provide a novel tool to treat diabetes and metabolic syndrome.

\author{
Abbreviations \\ PACAP: Pituitary adenylate cyclase-activating polypeptide \\ VIP: Vasoactive intestinal peptide \\ HFD: High-fat diet \\ NCD: Normal chow diet \\ LAR: Leukocyte common antigen-related \\ PTPRF: Receptor type protein tyrosine phosphatase F \\ GTT: Glucose tolerance test \\ ITT: Insulin tolerance test.
}

\section{Competing Interests}

The authors of this manuscript have no conflict of interests to declare.

\section{Acknowledgments}

This study was supported by CREST, JST, to MK. A part of this study was supported by Grant-in-Aid for Scientific Research (C) (JP24592277 and JP15K09442 to Masanori Nakata), for Challenging Exploratory Research (JP26670453 to Toshihiko Yada and JP26670122 to Hitoshi Hashimoto), for Scientific Research (B) (JP26293020 to Hitoshi Hashimoto), and for Scientific Research on Innovative Areas (JP15H01288 to Hitoshi Hashimoto) from Japan Society for the Promotion of Science (JSPS) and grants from Japan Diabetes Foundation to Masanori Nakata and Toshihiko Yada. A part of this work was supported by Ministry of Education, Culture, Sports, Science and Technology of Japan (MEXT) Supported Programs for Strategic Research Foundation at Private Universities 20112015 and 2013-2017 and a grant for Joint Researches in National Institute for Physiological Sciences to Toshihiko Yada. This study was subsidized by JKA through its promotion funds from KEIRIN RACE to Toshihiko Yada. A part of this work was also supported by the JSPS Program for Advancing Strategic International Networks to Accelerate the Circulation of Talented Researchers, Grant no. S2603 and the SRPBS from AMED to Hitoshi Hashimoto.

\section{References}

[1] T. Yada, M. Sakurada, K. Ihida et al., "Pituitary adenylate cyclase activating polypeptide is an extraordinarily potent intrapancreatic regulator of insulin secretion from islet $\beta$-cells," The Journal of Biological Chemistry, vol. 269, no. 2, pp. 1290-1293, 1994.

[2] T. Yada, M. Sakurada, H. Ishihara et al., "Pituitary adenylate cyclase-activating polypeptide (PACAP) is an islet substance serving as an intra-islet amplifier of glucose-induced insulin secretion in rats," Journal of Physiology, vol. 505, no. 2, pp. 319328, 1997.

[3] M. Nakata, S. Shioda, Y. Oka, I. Maruyama, and T. Yada, "Insulinotropin PACAP potentiates insulin-stimulated glucose uptake in 3T3 L1 cells," Peptides, vol. 20, no. 8, pp. 943-948, 1999. 
[4] M. Nakata, D. Kohno, N. Shintani et al., "PACAP deficient mice display reduced carbohydrate intake and PACAP activates NPYcontaining neurons in the rat hypothalamic arcuate nucleus," Neuroscience Letters, vol. 370, no. 2-3, pp. 252-256, 2004.

[5] M. J. Krashes, B. P. Shah, J. C. Madara et al., "An excitatory paraventricular nucleus to AgRP neuron circuit that drives hunger," Nature, vol. 507, no. 7491, pp. 238-242, 2014.

[6] Y. Shiotani, S. Kimura, Y. Ohshige, C. Yanaihara, and N. Yanaihara, "Immunohistochemical localization of pituitary adenylate cyclase-activating polypeptide (PACAP) in the adrenal medulla of the rat," Peptides, vol. 16, no. 6, pp. 1045-1050, 1995.

[7] D. Vaudry, B. J. Gonzalez, M. Basille, L. Yon, A. Fournier, and H. Vaudry, "Pituitary adenylate cyclase-activating polypeptide and its receptors: from structure to functions," Pharmacological Reviews, vol. 52, no. 2, pp. 269-324, 2000.

[8] A. Arimura, A. Somogyvári-Vigh, A. Miyata, K. Mizuno, D. H. Coy, and C. Kitada, "Tissue distribution of PACAP as determined by RIA: highly abundant in the rat brain and testes," Endocrinology, vol. 129, no. 5, pp. 2787-2789, 1991.

[9] G. Légrádi, S. Shioda, and A. Arimura, "Pituitary adenylate cyclase-activating polypeptide-like immunoreactivity in autonomic regulatory areas of the rat medulla oblongata," Neuroscience Letters, vol. 176, no. 2, pp. 193-196, 1994.

[10] D. Spengler, C. Waeber, C. Pantaloni et al., "Differential signal transduction by five splice variants of the PACAP receptor," Nature, vol. 365, no. 6442, pp. 170-175, 1993.

[11] A. J. Harmar, J. Fahrenkrug, I. Gozes et al., "Pharmacology and functions of receptors for vasoactive intestinal peptide and pituitary adenylate cyclase-activating polypeptide: IUPHAR Review 1," British Journal of Pharmacology, vol. 166, no. 1, pp. 4-17, 2012.

[12] T. K. Chatterjee, R. V. Sharma, and R. A. Fisher, "Molecular cloning of a novel variant of the pituitary adenylate cyclaseactivating polypeptide (PACAP) receptor that stimulates calcium influx by activation of L-type calcium channels," Journal of Biological Chemistry, vol. 271, no. 50, pp. 32226-32232, 1996.

[13] L. Åkesson, B. Ahrén, G. Edgren, and E. Degerman, "VPAC2$\mathrm{R}$ mediates the lipolytic effects of pituitary adenylate cyclaseactivating polypeptide/vasoactive intestinal polypeptide in primary rat adipocytes," Endocrinology, vol. 146, no. 2, pp. 744-750, 2005.

[14] S. Tomimoto, T. Ojika, N. Shintani et al., "Markedly reduced white adipose tissue and increased insulin sensitivity in Adcyap1-deficient mice," Journal of Pharmacological Sciences, vol. 107, no. 1, pp. 41-48, 2008.

[15] C. Yokota, K. Kawai, S. Ohashi, Y. Watanabe, and K. Yamashita, "PACAP stimulates glucose output from the perfused rat liver," Peptides, vol. 16, no. 1, pp. 55-60, 1995.

[16] Y. Sekiguchi, K. Kasai, K. Hasegawa, Y. Suzuki, and S.-I. Shimoda, "Glycogenolytic activity of Pituitary Adenylate Cyclase Activating Polypeptide (PACAP) in vivo and in vitro," Life Sciences, vol. 55, no. 15, pp. 1219-1228, 1994.

[17] M. Nakata, S. Yamamoto, T. Okada et al., "IL-10 gene transfer upregulates arcuate POMC and ameliorates hyperphagia, obesity and diabetes by substituting for leptin," International Journal of Obesity, vol. 40, no. 3, pp. 425-433, 2016.

[18] M. Nakata, T. Okada, K. Ozawa, and T. Yada, "Resistin induces insulin resistance in pancreatic islets to impair glucose-induced insulin release," Biochemical and Biophysical Research Communications, vol. 353, no. 4, pp. 1046-1051, 2007.

[19] M. Nakata, D. Gantulga, P. Santoso et al., "Paraventricular NUCB2/Nesfatin-1 supports oxytocin and vasopressin neurons to control feeding behavior and fluid balance in male mice," Endocrinology, vol. 157, no. 6, pp. 2322-2332, 2016.

[20] E. Li, M. Nakata, A. Shinozaki, Y. Yang, B. Zhang, and T. Yada, "Betatrophin expression is promoted in obese hyperinsulinemic type 2 but not type 1 diabetic mice," Endocrine Journal, vol. 63, no. 7, pp. 611-619, 2016.

[21] M. Kasuga, F. A. Karlsson, and C. R. Kahn, "Insulin stimulates the phosphorylation of the 95,000-dalton subunit of its own receptor," Science, vol. 215, no. 4529, pp. 185-187, 1982.

[22] D. Popov, "Novel protein tyrosine phosphatase $1 \mathrm{~B}$ inhibitors: interaction requirements for improved intracellular efficacy in type 2 diabetes mellitus and obesity control," Biochemical and Biophysical Research Communications, vol. 410, no. 3, pp. 377381, 2011.

[23] F. Ahmad and B. J. Goldstein, "Functional association between the insulin receptor and the transmembrane protein-tyrosine phosphatase LAR in intact cells," The Journal of Biological Chemistry, vol. 272, no. 1, pp. 448-457, 1997.

[24] F. Ahmad, J. L. Azevedo Jr., R. Cortright, G. L. Dohm, and B. J. Goldstein, "Alterations in skeletal muscle protein-tyrosine phosphatase activity and expression in insulin-resistant human obesity and diabetes," The Journal of Clinical Investigation, vol. 100, no. 2, pp. 449-458, 1997.

[25] F. Ahmad, R. V. Considine, T. L. Bauer, J. P. Ohannesian, C. C. Marco, and B. J. Goldstein, "Improved sensitivity to insulin in obese subjects following weight loss is accompanied by reduced protein-tyrosine phosphatases in adipose tissue," Metabolism: Clinical and Experimental, vol. 46, no. 10, pp. 1140-1145, 1997.

[26] P.-M. Li, W.-R. Zhang, and B. J. Goldstein, "Suppression of insulin receptor activation by overexpression of the proteintyrosine phosphatase LAR in hepatoma cells," Cellular Signalling, vol. 8, no. 7, pp. 467-473, 1996.

[27] A. T. Cheung, J. Wang, D. Ree, J. K. Kolls, and M. Bryer-Ash, "Tumor necrosis factor- $\alpha$ induces hepatic insulin resistance in obese Zucker ( $\mathrm{fa} / \mathrm{fa}$ ) rats via interaction of leukocyte antigenrelated tyrosine phosphatase with focal adhesion kinase," Diabetes, vol. 49, no. 5, pp. 810-819, 2000.

[28] O. M. Finucane, C. M. Reynolds, F. C. McGillicuddy, and H. M. Roche, "Insights into the role of macrophage migration inhibitory factor in obesity and insulin resistance," Proceedings of the Nutrition Society, vol. 71, no. 4, pp. 622-633, 2012.

[29] K. Moller, Y.-Z. Zhang, R. Håkanson et al., "Pituitary adenylate cyclase activating peptide is a sensory neuropeptide: immunocytochemical and immunochemical evidence," Neuroscience, vol. 57, no. 3, pp. 725-732, 1993.

[30] J. Fahrenkrug and J. Hannibal, "PACAP in visceral afferent nerves supplying the rat digestive and urinary tracts," Annals of the New York Academy of Sciences, vol. 865, pp. 542-546, 1998.

[31] K. M. Braas and V. May, "Pituitary adenylate cyclase-activating polypeptides directly stimulate sympathetic neuron neuropeptide $\mathrm{Y}$ release through $\mathrm{PACl}$ receptor isoform activation of specific intracellular signaling pathways," Journal of Biological Chemistry, vol. 274, no. 39, pp. 27702-27710, 1999.

[32] C. A. Brandenburg, V. May, and K. M. Braas, "Identification of endogenous sympathetic neuron pituitary adenylatecyclaseactivating polypeptide (PACAP): depolarization regulates production and secretion through induction of multiple propeptide transcripts," Journal of Neuroscience, vol. 17, no. 11, pp. 40454055, 1997. 
[33] K. Persson and B. Ahrén, "The neuropeptide PACAP contributes to the glucagon response to insulin-induced hypoglycaemia in mice," Acta Physiologica Scandinavica, vol. 175, no. 1, pp. 25-28, 2002.

[34] Y. Fukushima, T. Nagayama, H. Kawashima et al., "Role of calcium channels and adenylate cyclase in the PACAP-induced adrenal catecholamine secretion," American Journal of Physiology. Regulatory, Integrative and Comparative Physiology, vol. 28, no. 2, pp. R495-R501, 2001.

[35] X. Liu, Q. Xiao, L. Zhang et al., "The long-term efficacy and safety of DPP-IV inhibitors monotherapy and in combination with metformin in 18980 patients with type-2 diabetes mellitusa meta-analysis," Pharmacoepidemiology and Drug Safety, vol. 23, no. 7, pp. 687-698, 2014.

[36] L. Zhu, C. Tamvakopoulos, D. Xie et al., "The role of dipeptidyl peptidase IV in the cleavage of glucagon family peptides: in vivo metabolism of pituitary adenylate cyclase-activating polypeptide-(1-38)," The Journal of Biological Chemistry, vol. 278, no. 25, pp. 22418-22423, 2003.

[37] B. Ahrén and T. E. Hughes, "Inhibition of dipeptidyl peptidase4 augments insulin secretion in response to exogenously administered glucagon-like peptide-1, glucose-dependent insulinotropic polypeptide, pituitary adenylate cyclase-activating polypeptide, and gastrin-releasing peptide in mice," Endocrinology, vol. 146, no. 4, pp. 2055-2059, 2005.

[38] M. Tsutsumi, T. H. Claus, Y. Liang et al., "A potent and highly selective VPAC2 agonist enhances glucose-induced insulin release and glucose disposal: a potential therapy for type 2 diabetes," Diabetes, vol. 51, no. 5, pp. 1453-1460, 2002.

[39] J. Kosacka, T. Schröder, I. Bechmann et al., "PACAP upregulates the expression of apolipoprotein $\mathrm{D}$ in 3T3-L1 adipocytes. DRG/3T3-L1 co-cultures study," Neuroscience Research, vol. 69 , no. 1, pp. 8-16, 2011.

[40] H. R. Lijnen, K. Freson, and M. F. Hoylaerts, "Effect of VPAC1 blockade on adipose tissue formation and composition in mouse models of nutritionally induced obesity," Journal of Obesity, vol. 2010, Article ID 359527, 5 pages, 2010. 


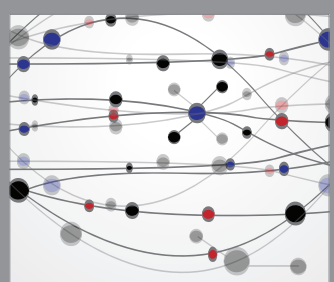

The Scientific World Journal
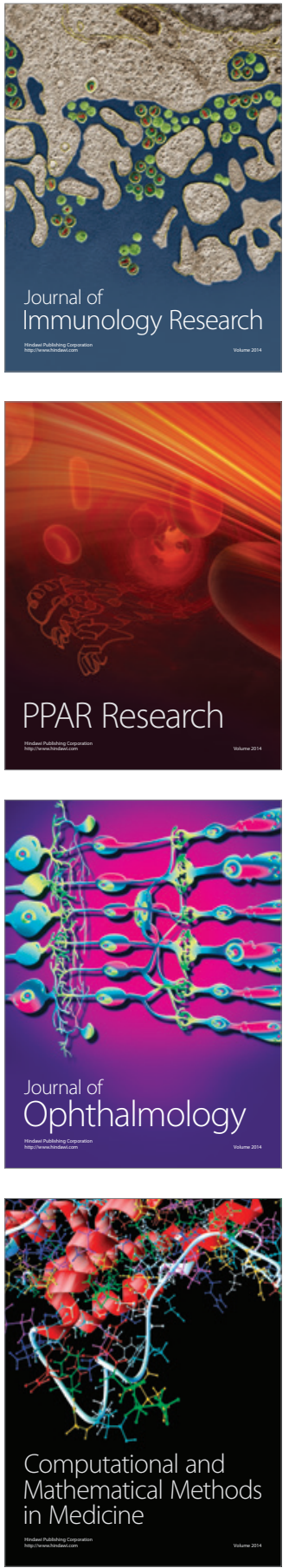

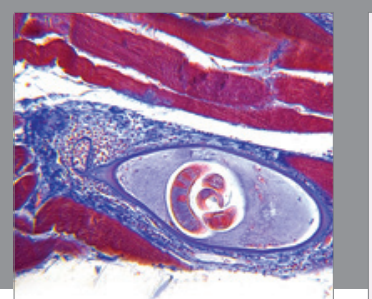

Gastroenterology Research and Practice

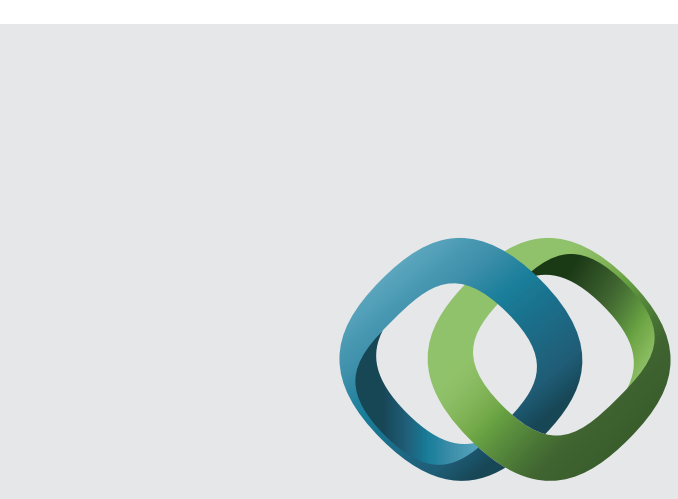

\section{Hindawi}

Submit your manuscripts at

http://www.hindawi.com
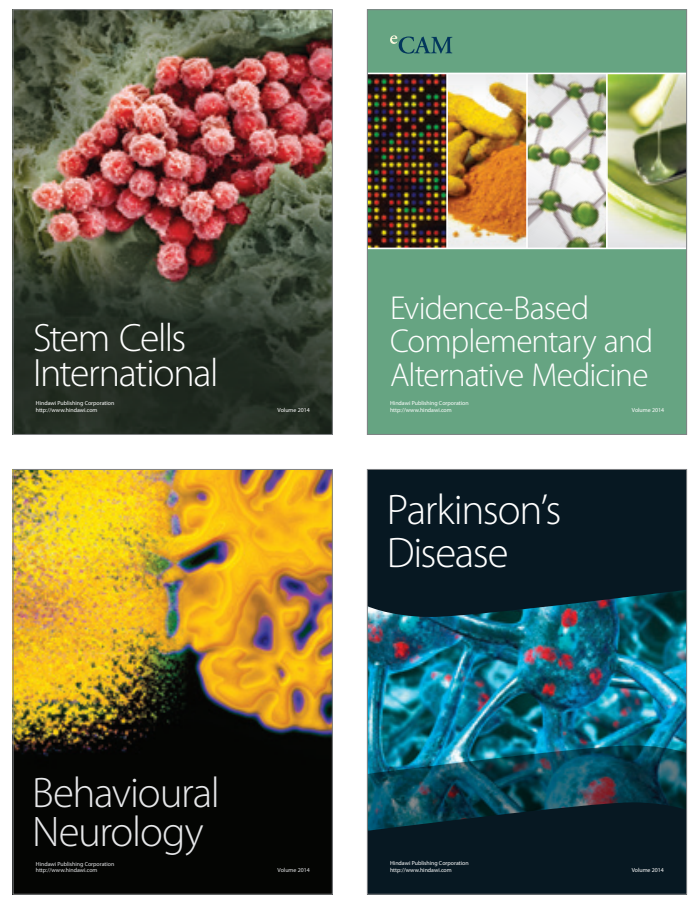
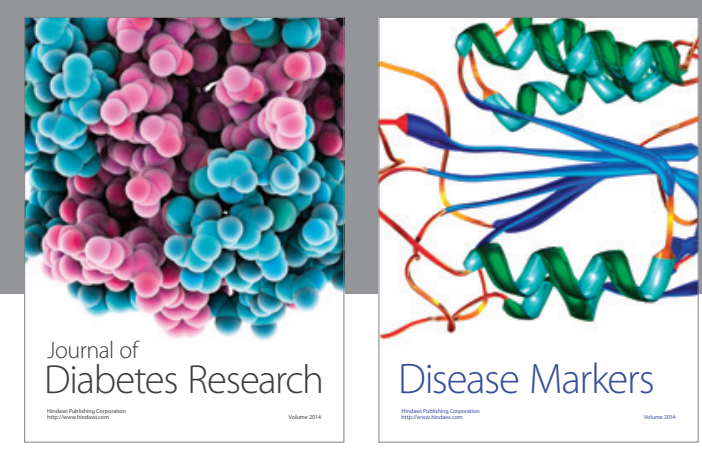

Disease Markers
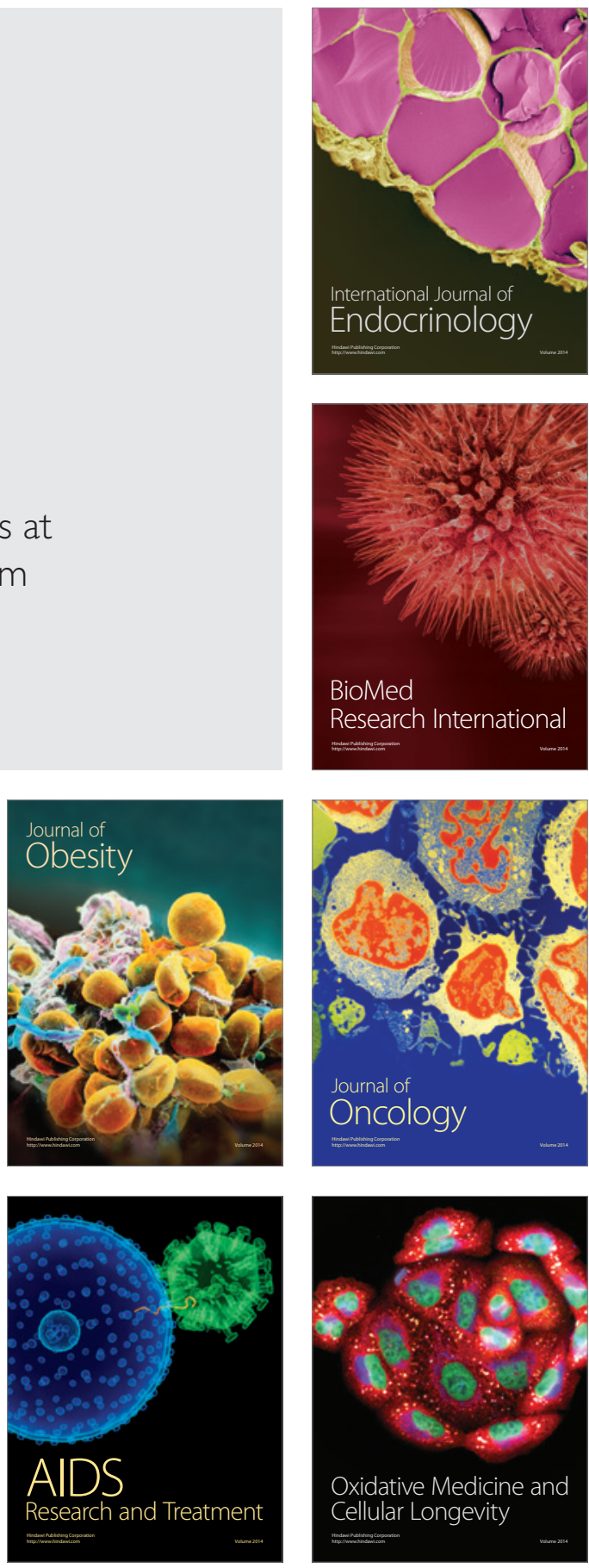